\title{
Study of CMOS strip sensor for future silicon tracker
}

Y. Han ${ }^{\mathrm{r}, \mathrm{t}, *}, \mathrm{H}$. Zhu $^{\mathrm{r}, \mathrm{u}, * *}$, A. Affolder ${ }^{\mathrm{i}}$, K. Arndt ${ }^{\mathrm{b}}$, R. Bates ${ }^{\mathrm{c}}$, M. Benoit ${ }^{\mathrm{d}}$, F. Di Bello ${ }^{\mathrm{d}}$, A. Blue ${ }^{\mathrm{c}}$, D. Bortoletto $^{b}$, M. Buckland ${ }^{\mathrm{a}, \mathrm{e}}$, C. Buttar ${ }^{\mathrm{c}}$, P. Caragiulo $^{\mathrm{f}}$, Y. Chen ${ }^{\mathrm{r}, \mathrm{t}}$, D. Das ${ }^{\mathrm{g}}$, D. Doering ${ }^{\mathrm{f}}$, J. Dopkes ${ }^{g}$, A. Dragone ${ }^{\mathrm{f}}$, F. Ehrler ${ }^{\mathrm{h}}$, V. Fadeyev ${ }^{\mathrm{i}}$, W. Fedorko ${ }^{\mathrm{s}}$, Z. Galloway ${ }^{\mathrm{i}}$, C. Gay ${ }^{\mathrm{s}}$, H. Grabas ${ }^{i}$, I.M. Gregor ${ }^{\mathrm{j}}$, P. Grenier ${ }^{\mathrm{f}}$, A. Grillo ${ }^{\mathrm{i}}$, B. Hiti ${ }^{\mathrm{m}}$, M. Hoeferkamp ${ }^{\mathrm{k}}$, L.B.A. Hommels ${ }^{1}$, T. Huffman ${ }^{\text {b }}$, J. John ${ }^{\text {b }}$, K. Kanisauskas ${ }^{\text {b,c }}$, C. Kenney ${ }^{\text {f }}$, G. Kramberger ${ }^{\text {m }}$, P. Liu ${ }^{\text {r,u }}$, W. Lu ${ }^{\text {r,u }}$, Z. Liang ${ }^{\mathrm{r}}$, I. Mandić ${ }^{\mathrm{m}}$, D. Maneuski ${ }^{\mathrm{c}}$, F. Martinez-Mckinney ${ }^{\mathrm{i}}$, S. McMahon ${ }^{\mathrm{b}, \mathrm{g}}$, L. Meng a,d, M. Mikuž ${ }^{\mathrm{m}, \mathrm{n}}$, D. Muenstermann ${ }^{\mathrm{o}}$, R. Nickerson ${ }^{\mathrm{b}}$, I. Peric ${ }^{\mathrm{h}}$, P. Phillips ${ }^{\mathrm{b}, \mathrm{g}}$, R. Plackett ${ }^{\mathrm{b}}$, F. Rubbo ${ }^{\mathrm{f}}$, L. Ruckman ${ }^{\text {f }}$, J. Segal ${ }^{\text {f }}$, S. Seidel ${ }^{\text {k }}$, A. Seiden ${ }^{i}$, I. Shipsey ${ }^{\text {b }}$, W. Song ${ }^{\mathrm{r}}$, M. Stanitzki ${ }^{\mathrm{j}}$, D. Su $^{\mathrm{f}}$, C. Tamma ${ }^{\text {f }}$, R. Turchetta ${ }^{g}$, L. Vigani ${ }^{\text {b }}$, J. Volk ${ }^{i}$, R. Wang ${ }^{\mathrm{p}}$, M. Warren ${ }^{\mathrm{q}}$, F. Wilson ${ }^{\mathrm{g}}$, S. Worm ${ }^{\mathrm{g}}$, Q. Xiu ${ }^{\mathrm{r}}$, J. Zhang ${ }^{\mathrm{p}}$

a University of Liverpool, United Kingdom

${ }^{\mathrm{b}}$ University of Oxford, United Kingdom

${ }^{\mathrm{c}}$ SUPA - School of Physics and Astronomy, University of Glasgow, Glasgow, United Kingdom

d University of Geneva, Switzerland

e CERN, European Center for Nuclear Research, Switzerland

${ }^{\mathrm{f}}$ SLAC National Accelerator Laboratory, United States

${ }^{g}$ Rutherford Appleton Laboratory, Didcot, United Kingdom

${ }^{\mathrm{h}}$ Karlsruhe Institute of Technology, Germany

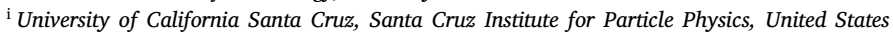

j Deutsches Elektronen-Synchrotron, Germany

${ }^{\mathrm{k}}$ University of New Mexico, United States

${ }^{1}$ Cambridge University, United Kingdom

${ }^{\mathrm{m}}$ Joẑef Stefan Institute, Ljubljana, Slovenia

${ }^{\mathrm{n}}$ University of Ljubljana, Slovenia

${ }^{\circ}$ Lancaster University, United Kingdom

${ }^{\mathrm{p}}$ Argonne National Laboratory, United states

${ }^{\mathrm{q}}$ University College, London, United Kingdom

${ }^{\mathrm{r}}$ Institute of High Energy Physics, Beijing, China

${ }^{s}$ University of British Columbia, Canada

${ }^{\mathrm{t}}$ University of Chinese Academy of Sciences, Beijing, China

"State Key Laboratory of Particle Detection and Electronics, Beijing, China

\section{A R T I C L E I N F O}

Keywords:

Monolithic silicon sensor

High-Voltage CMOS

CHESS

Large-area tracking system

\begin{abstract}
A B S T R A C T
Monolithic silicon sensors developed with High-Voltage CMOS (HV-CMOS) processes have become highly attractive for charged particle tracking. Compared with the standard CMOS sensors, HV-CMOS sensors can provide larger and deeper depletion regions that lead to larger signals and faster charge collection. They can provide high position resolution, low material budget, high radiation hardness and low cost that are desirable for high performance tracking in harsh collision environment. Various studies have been conducted to explore the technology feasibility for the large-area tracking systems at future collider experiments.

CHESS (CMOS HV/HR Evaluation for Strip Sensor) sensor series have been developed as an alternative solution to the conventional silicon micro-strip detectors for the ATLAS inner tracker upgrade. The first prototype (named CHESS1) was to evaluate the diode geometry and the in-pixel analog electronics. Obtained test results were used to optimize the second prototype (named CHESS2). CHESS2 was implemented with a full digital readout architecture and realized as a full reticle sized monolithic sensor. In this paper, the basic characteristics of the CHESS2 prototype sensors and their performance in response to different input signals are presented.
\end{abstract}

* Corresponding author at: Institute of High Energy Physics, Beijing, China.

** Corresponding author at: Institute of High Energy Physics, Beijing, China. E-mail addresses: hanyubo@ihep.ac.cn (Y. Han), zhuhb@ihep.ac.cn (H. Zhu). 


\section{Introduction}

Silicon detectors have been widely used in the tracking systems at high energy physics experiments for their proficient capability of high position resolution, fast readout and high radiation tolerance. In silicon detectors, electron-hole pairs are generated instantaneously along the trajectory of the impinging charged particle that transverses the sensitive volume of the device. These charge carriers are then collected by the embedded electrodes via the drift and/or diffusion processes. The induced current on the electrode can be transmitted to a readout circuit for further signal processing. In conventional silicon detectors, the detection sensor and its readout electronics are implemented in separated devices and electrically connected via wire-bonding or bump-bonding. For large area tracking systems, such inter-connection processes can be rather expensive and time consuming. Monolithic Active Pixel Sensors (MAPS) can integrate both sensing element and readout electronics onto the same silicon substrate without any additional complicated inter-connection process. They were suggested for charged particle tracking [1] and already deployed for the PiXeL detector (PXL) of the STAR experiment at RHIC [2] as well as the more recent ALICE ITS Upgrade $[3,4]$. They have been also considered for tracking systems at several future collider experiments [5-7].

The standard MAPS sensors typically develop a shallow depletion region and can collect charge carriers mostly via diffusion. Silicon sensors fabricated with the High-Voltage CMOS (HV-CMOS) technology can form a deeper depletion region with an external bias voltage, and collect charge carriers via drift that can lead to a shorter collection time and improved radiation hardness [8]. In addition, the selected AMS HVCMOS technology allows the usage of high bulk-resistivity wafers that can increase more the depletion region. These features make HV-CMOS sensors extremely attractive for the large-area silicon tracking systems at future collider experiments.

CHESS (CMOS HV/HR Evaluation for Strip Sensor) sensors were initially designed to evaluate the HV-CMOS detector concept as an alternative solution to the conventional micro-strip detector for the ATLAS inner tracker upgrade. Diode geometry and in-pixel analog electronics designs were verified with the first prototype CHESS1 and an earlier investigation sensor of HVStripV1 [9-11]. Test results showed that the sensors could withstand the radiation levels expected for the out tracker of the ATLAS inner tracker upgrade [12]. The standalone built-in amplifier was also been characterized [13]. With experience learnt and designs inherited from CHESS1, a second prototype in full reticle size, named CHESS2, was developed with complex in-pixel electronics and a full digital readout architecture. In this article, latest test results obtained from the sensor characterization using laser and radioactive source are reported.

\section{CHESS2 design}

CHESS2 was designed as a full reticle-sized monolithic HV-CMOS sensor with complex in-pixel electronics and a full digital readout architecture. It was fabricated with the AMS-H35 High-Voltage CMOS process and different bulk-resistivity wafers. The sensor can withstand an external bias voltage up to $120 \mathrm{~V}$. The prototype is $18.6 \times 24.3 \mathrm{~mm}^{2}$ in size and sensors are thinned down to $250 \mu \mathrm{m}$. As shown in Fig. 1, the prototype sensor consists of three main pixel arrays and each contains $40 \times 630 \mu \mathrm{m}^{2}$ sized pixels arranged in 128 rows and 32 columns with full digital encoding and readout. There are also passive pixels arranged in 16 rows and 32 columns implemented with multiplexing readout. There are test structures reserved for the edge-TCT scans and they are located at the edge of the middle section. Tests with the large passive pixel array were already performed to investigate the radiation hardness of the sensor and results were published in Refs. [10,12].

In the three main pixel arrays, each pixel contains six interconnected NWELLs of $24.3 \times 80.2 \mu \mathrm{m}^{2}$ in size. As illustrated in Fig. 2, the in-pixel electronics is located in the area of the two leftmost

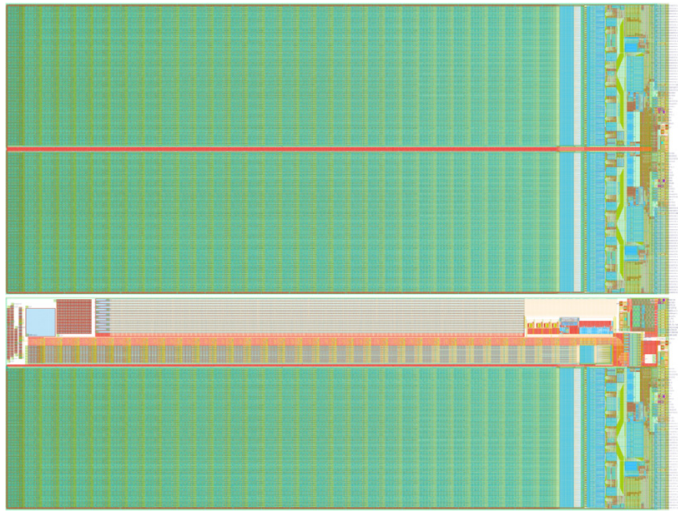

Fig. 1. Layout of the CHESS2 prototype sensor.

NWELLs and contains a charge sensitive amplifier, an RC-CR shaper, a 4-bit DAC for offset compensation, a comparator and a programmable charge injection circuit. The schematic of the in-pixel electronics is shown in Fig. 3. Both threshold and charge injection are common to all the pixels in each pixel array and set through the external pads. Pixels are programmed through a set of dedicated commands using the SACI interface. The built-in amplifiers are continuously sensitive and their outputs are discriminated and latched in each cycle of the $40 \mathrm{MHz}$ clock. With a latency of 2 clock cycles, the address of the first (left to right) hit in a row of pixels is encoded into 5 bits. Additional hits along the pixel row set a 6th bit. In each cycle of the $40 \mathrm{MHz}$ clock, the 128 encoded hit values are sent to the pixel array encoder which selects the first (top to bottom) 8 hits within a latency of 2 clock cycles. The addresses of the 8 hits are serialized on 13 LVDS pairs at the clock frequency of $320 \mathrm{MHz}$. More design details can be found in Ref. [14]. Table 1 summarizes the main design differences between CHESS 1 and CHESS2.

\section{Test setup}

The test system consists of a daughter board carrying a CHESS2 sensor and a carrier board with a commercial Xilinx FPGA. The carrier board receives and processes the data from the daughter board before sending them out to the controller PC via Ethernet. It also powers the sensor under test and provides charge injection pulses for in-pixel circuit calibration. The data acquisition software is based on Rogue, ${ }^{1}$ which is a Python based hardware abstraction and data acquisition system developed at SLAC. CHESS2 can operate in either the register reading mode or the stream readout mode. In the register reading mode (debugging mode), it first sends out a width-controllable square pulse and opens a length programmable time window at the same time that allows to record the first 8 hits latched in memory. In this operation mode, not only the addresses of the fired pixels, but also the arrival time of each registered hit recorded with a precision of $\mathcal{O}(\mathrm{ns})$ are read out. In the stream readout mode, which is used for the standard detector operation, upon receiving an external trigger, data packs with a selectable size up to 256 bytes are read out. Both readout modes have been verified and demonstrated to provide stable and reliable data taking.

The experimental setup for the laser test is depicted in Fig. 4. The daughter board and the carrier board were fixed to an Aluminum plate, which was mounted on a movable $x-y$ stage with high precision. The daughter board was aligned perpendicular to the laser beam and moved to the focal plane to minimize the beam spot size at the sensor surface. The whole system was placed inside an Aluminum shielding box. The setup for the radioactive source test was similar but with a CHESS2 sensor facing downwards and a Fe-55 source fixed right underneath.

\footnotetext{
1 Accessible at https://github.com/slaclab/rogue
} 


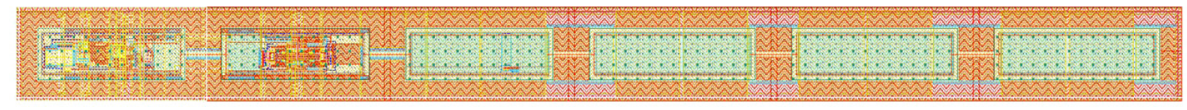

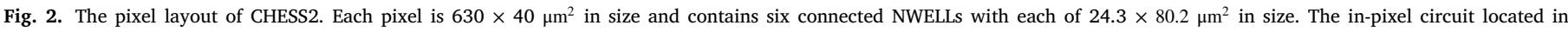
the left two NWELLs includes amplifier, comparator, charge injection, pixel selection and comparator trimming memory.

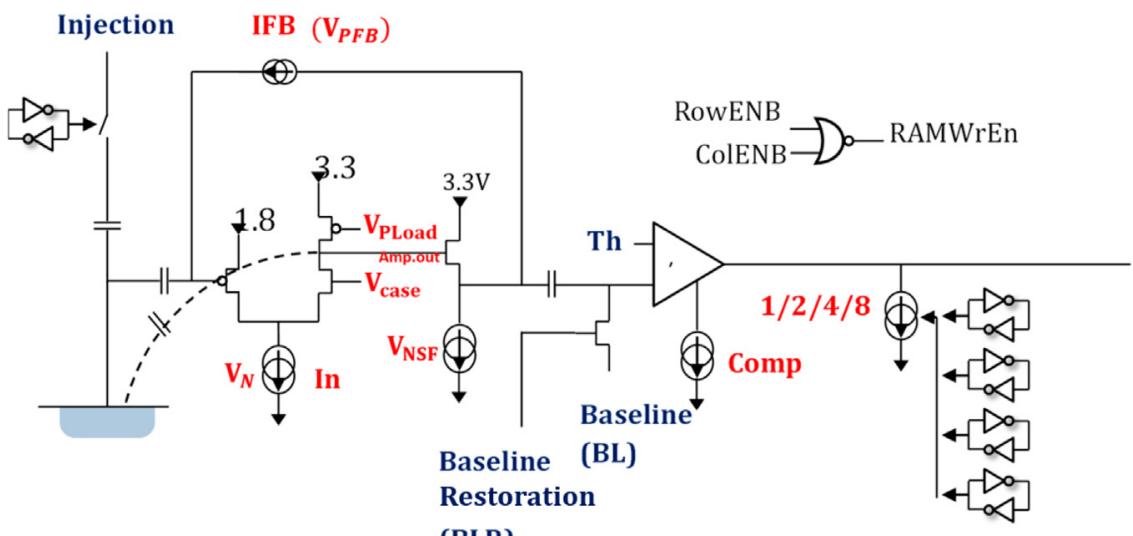

(BLR)

Fig. 3. Schematic of the electronics in the pixels.

Table 1

Main design differences between CHESS1 and CHESS2.

\begin{tabular}{lll}
\hline & CHESS1 & CHESS2 \\
\hline Sensor size & $3.4 \times 4.2 \mathrm{~mm}^{2}$ & $18.6 \times 24.3 \mathrm{~mm}^{2}$ \\
\hline $\begin{array}{l}\text { Substrate } \\
\text { resistivity }\end{array}$ & Default $20 \mathrm{Ohm} \cdot \mathrm{cm}$ & $\begin{array}{l}\text { Four values between } 20 \text { and } \\
2000 \mathrm{Ohm} \cdot \mathrm{cm}\end{array}$ \\
\hline Pixel size & $\begin{array}{l}\text { Passive: } 45 \times 100-800 \mu \mathrm{m}^{2}, \text { with } \\
\text { varying diode area; Active: }\end{array}$ & $40 \times 600 \mu \mathrm{m}^{2}$ \\
& $5 \times 200 \mu \mathrm{m}^{2}$ & \\
\hline Design emphasis & $\begin{array}{l}\text { Basic building blocks: passive } \\
\text { diodes, stand-alone amplifiers, } \\
\text { transistors, small number of } \\
\text { active pixels }\end{array}$ & $\begin{array}{l}\text { Full reticle size: large pixel } \\
\text { matrices of } 128 \text { by } 32 \text { pixels } \\
\text { each, full digital readout } \\
\text { architecture }\end{array}$ \\
\hline $\begin{array}{l}\text { In-pixel } \\
\text { electronics }\end{array}$ & $\begin{array}{l}\text { Re-optimized and added: } \\
\text { charge injection, tunable bias } \\
\text { the HVStripV1 design [10] }\end{array}$ & $\begin{array}{l}\text { currents, threshold tunable } \\
\text { discriminators, etc. }\end{array}$ \\
\hline
\end{tabular}

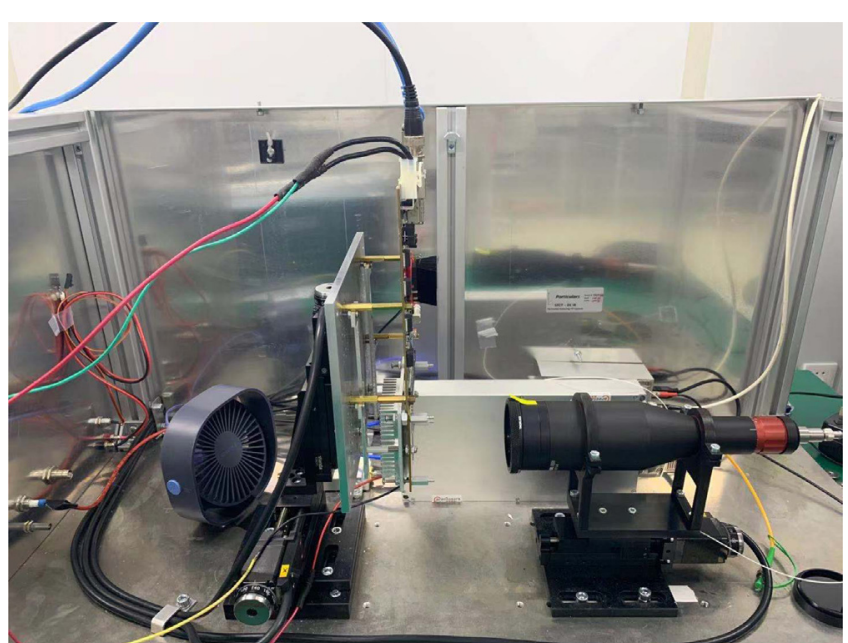

Fig. 4. Both daughter board and carrier board were mounted on a supporting plated fixed to the $x-y$ stage and the daughter board was aligned perpendicular to the laser beam.

\section{Test results}

\subsection{Scan and in-pixel circuit tuning}

Leakage current of the active matrix arrays was measured for several CHESS2 prototype sensors with different substrate resistivities. Obtained results were summarized in Fig. 5(a). High leakage current was observed in all the tested sensors, among which only sensors with the substrate resistivity of $200 \mathrm{Ohm} \cdot \mathrm{cm}$ could still hold the leakage current below $1 \mathrm{~mA}$ after depletion. The linear portion of the IV curve was due to a $10 \mathrm{kOhm}$ resistor implemented in series with the sensor. Sensors with the $20 \mathrm{Ohm} \cdot \mathrm{cm}$ substrate resistivity encountered early breakdown that led to the extended linear portion of the measured IV curves. Connections between the carrier board and the daughter were fully checked but nothing that could cause the large current. Such large leakage current might be due to the absence of a guard ring surrounding the pixel array, and/or the parasitic coupling between the sensor and its digital circuit, and subject to further investigation. Based on the IV scan results, prototype sensors with the $200 \mathrm{Ohm} \cdot \mathrm{cm}$ substrate resistivity were chosen for further characterization tests.

To derive the proper range of the baseline and the threshold parameters, threshold sweeping at different baseline levels was performed for each individual pixel. Using the register reading mode, results with 


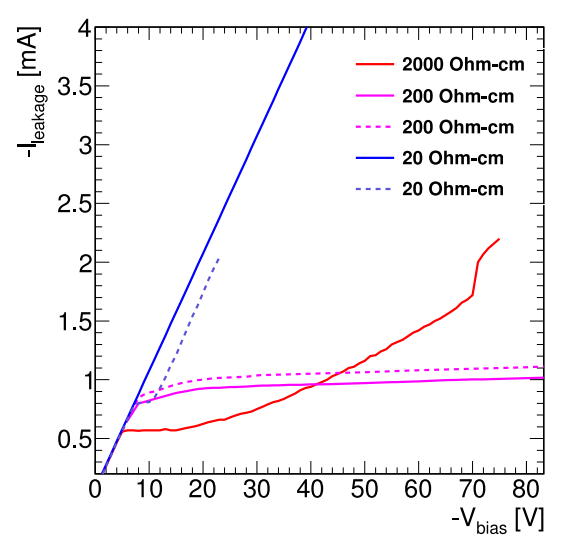

(a)

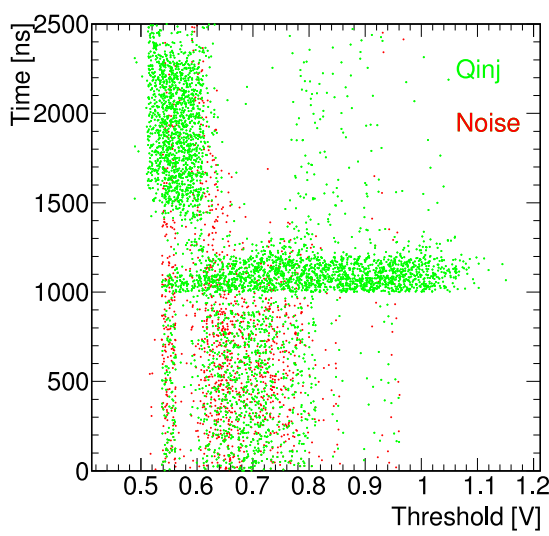

(b)

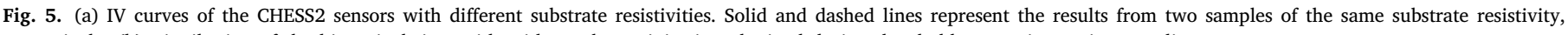
respectively. (b) Distribution of the hit arrival time with/without charge injection obtained during threshold scan using register reading.

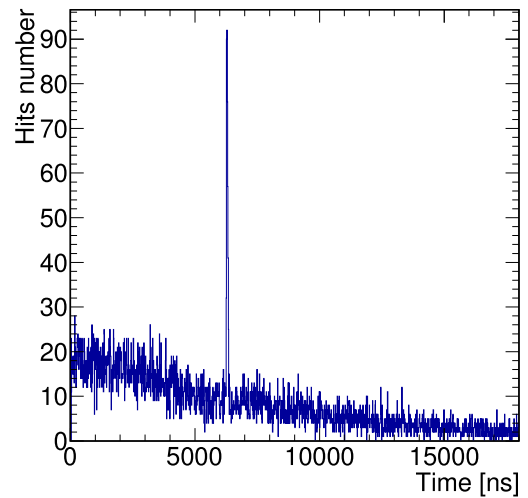

(a) Time distribution

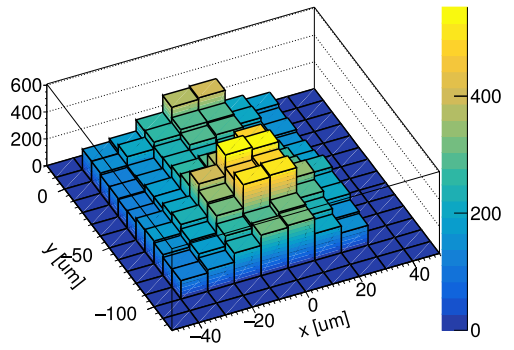

(b) Scan of one diode

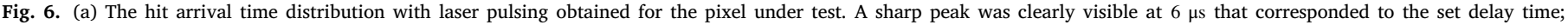
(b) Hit counts obtained for different $(x, y)$ positions from the pixel scan.

charge injection (Qinj) on and off at different baseline levels were obtained. A fixed delay of $1 \mu$ s to the charge injection was introduced to help distinguishing the signal from noise. Signal hits with the specific time information was clearly observed in Fig. 5(b). The results also suggested the working range between $0.6 \mathrm{~V}$ and $0.9 \mathrm{~V}$ for the baseline and threshold settings. During the tests, it was also noticed that temperature control was important to stabilize the sensor performance.

It should be noted that both baseline and threshold levels were set globally to all the pixels in the same array, but the actual value applied to each individual pixel might differ. To mitigate this effect, a dedicated threshold trimming circuit was implemented in each pixel to minimize the threshold variation. However, due to a design issue, this important trimming circuit did not function as expected. Consequently, the large variation in pixel thresholds prohibited the proper data taking for the whole pixel array. If several pixels were enabled and read out together, hits from pixels with lower thresholds could block completely the ones with higher thresholds. To continue the tests below, one single pixel was enabled at a time.

\subsection{Tests with laser}

The prototype sensor was first confirmed sensitive to LED light and could yield the correct arrival time information of the hits. Further tests were then performed with a red laser with a wavelength of $640 \mathrm{~nm}$. The red laser was tuned to be with a pulse width of $5 \mathrm{~ns}$ and focused to a beam spot of $\mathcal{O}(10) \mu \mathrm{m}$ in diameter at the sensor surface. The focusing was achieved by adjusting the distance between the laser and the sensor until the maximum number of hit counts within the set time window was obtained.

Using the register reading, a trigger signal was sent to the daughter board to enable the pixel data taking. A time delay of $6 \mu \mathrm{s}$ was introduced before firing the laser. The arrival time of the hits developed a peak at around $6 \mu \mathrm{s}$ as shown in Fig. 6(a), but broadened to about $200 \mathrm{~ns}$ due to the jitter on the triggering pulses and the intrinsic laser instability. The pixel layout was scanned by moving the sensor under test in both $\mathrm{x}$ and $\mathrm{y}$ directions. Fig. 6(b) shows the hit counts in a narrow time window around $6 \mu$ s for different $(x,(y)$ positions. The scan results showed a diode profile consistent with the pixel design shown in Fig. 2. The same structure was also confirmed with the stream readout mode.

Sensor response to the red laser was also tested with different bias voltage applied. The results were obtained by shooting the laser around the diode area with the bias voltage changed from 0 to $-120 \mathrm{~V}$. Fig. 7(a) shows a monotonic increase of the hit counts with higher bias voltage. The hit counts saturated around $-60 \mathrm{~V}$, as shown in Fig. $7(\mathrm{~b})$, due to the limited penetration capability of the red laser. The exact depletion depth might continue to increase beyond this voltage.

\subsection{Test with $\mathrm{Fe}-55$}

Tests with a Fe-55 radioactive source were also performed. The sensor was biased at $-40 \mathrm{~V}$ and one pixel was enabled for data 


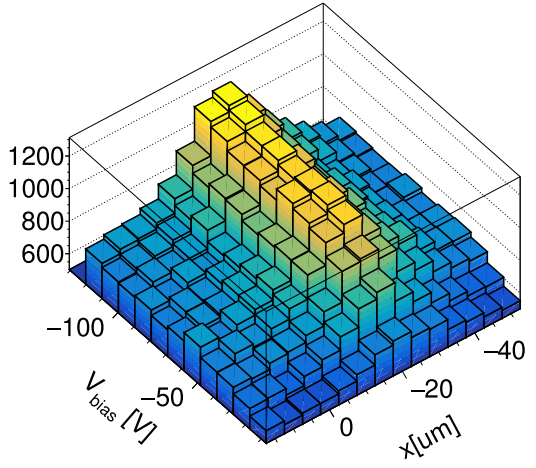

(a)

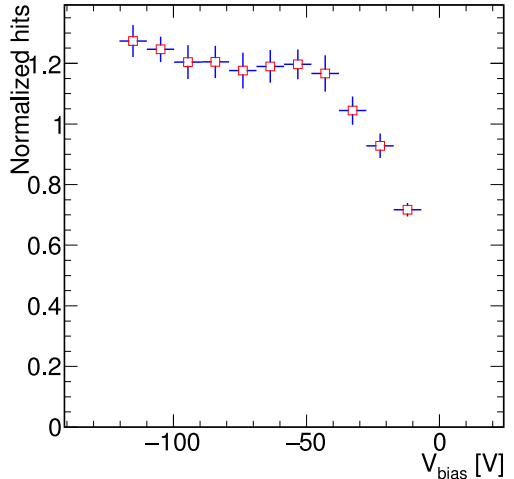

(b)

Fig. 7. (a) Hit counts at different positions along the pixel short end and the applied bias voltage; (b) Normalized hit counts as a function of the bias voltage.

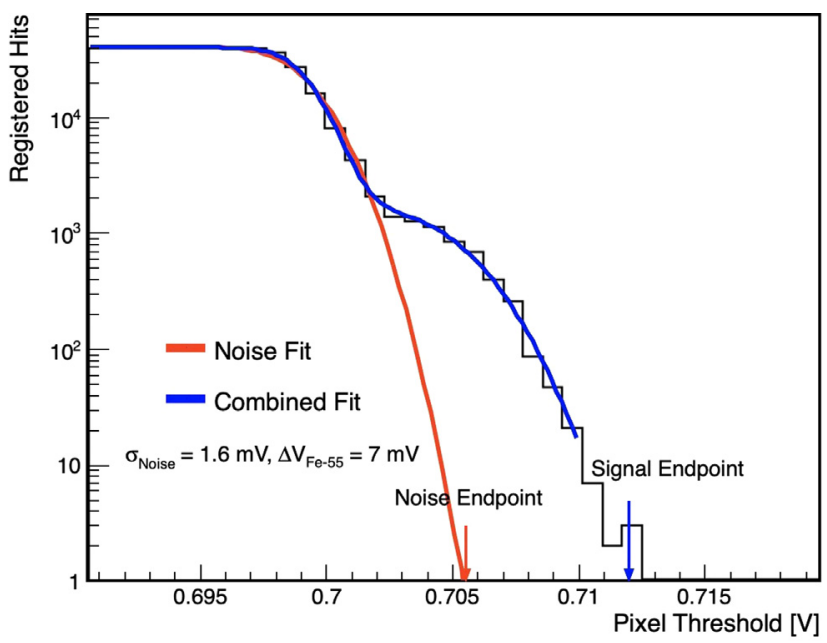

Fig. 8. Hit count distribution form the threshold scan with a Fe-55 radioactive source fitted with two combined error functions. The second but low plateau indicated the hits from the low energy X-ray signals.

taking. With the limited sensitive area of one single pixel and the low radioactivity of the source, the effective signal rate was rather low. Fig. 8 shows the hit counts obtained from the threshold scans in the range of $[0.68,0.72 \mathrm{~V}]$. The signal from the low energy X-ray (5.9 $\mathrm{keV}$ ) yielded a second but low plateau in the hit count distribution. A function constructed from two error functions, representing the noise and the signal components, were fit to the hit count distribution. The signal amplitude was estimated to be $7 \mathrm{mV}$, by taking the difference between the end-points of the noise and signal distributions. The noise level was estimated to be $1.6 \mathrm{mV}$ or an equivalent noise charge (ENC) of $385 \mathrm{e}^{-}$from the spread of the falling edge of the noise distribution. The noise level was significantly larger than the design expectation. The signal-to-noise ratio $(\mathrm{S} / \mathrm{N})$ was estimated to be about 4.3 . The relatively high noise level and low $\mathrm{S} / \mathrm{N}$ compared with the design expectations, might be due to the front-end amplifier changes, extra capacitance from the readout lines and other common mode effects, and should be investigated with more tests in future.

\section{Summary}

HV-CMOS technology has attracted increasing interests for the large-area silicon tracking systems at future collider experiments. CHESS2, which was a full reticle-sized sensor using the AMS-H35 HighVoltage CMOS process, was designed with complex in-pixel electronics and a full digital readout architecture to explore the technology feasibility. Prototype sensors were fabricated with wafers of different substrate resistivities. Despite of the unexpected large leakage current and the malfunctioning threshold trimming circuit, sensors were characterized with a red laser and a Fe-55 radioactive source. Results obtained with the laser scan showed the diode profile consistent with the pixel design. From the radioactive source tests, the equivalent noise charge was measured to be $385 \mathrm{e}^{-}$and the $\mathrm{S} / \mathrm{N}$ ratio was estimated as 4.3 . The relatively high noise level and low $\mathrm{S} / \mathrm{N}$ might be due to the front-end amplifier changes, extra capacitance from the readout lines and other common mode effects. Such effects should be investigated with more tests in future.

\section{Declaration of competing interest}

The authors declare that they have no known competing financial interests or personal relationships that could have appeared to influence the work reported in this paper.

\section{Acknowledgment}

The research was supported and financed by National Key Programme for S\&T Research and Development (Grant no. 2016YFA0400101) and the CAS International Partnership Program, UK Science and Technology Facilities Council (STFC), the Slovenian Research Agency, the United States Department of Energy, grant DESC0010107, and the SLAC LDRD program. The research leading to these results has received funding from the European Commission under the FP7 Research Infrastructures project AIDA, grant agreement no. 262025 .

\section{References}

[1] R. Turchetta, et al., A monolithic active pixel sensor for charged particle tracking and imaging using standard VLSI CMOS technology, Nucl. Instrum. Meth. 458 (3) (2001) 677-689, http://dx.doi.org/10.1016/S0168-9002(00)00893-7.

[2] L. Greiner, et al., A MAPS based vertex detector for the STAR experiment at RHIC, Nucl. Instrum. Meth. 650 (1) (2011) 68-72, http://dx.doi.org/10.1016/j. nima.2010.12.006

[3] B. Abelev, et al., Technical design report for the upgrade of the ALICE inner tracking system, J. Phys. G: Nucl. Part. Phys. 41 (8) (2014) 087002, http: //dx.doi.org/10.1088/0954-3899/41/8/087002.

[4] D. Colella, ALICE inner tracking system upgrade: construction and commissioning, 2019, arXiv:1912.12188.

[5] Y. Zhang, et al., Charge collection and non-ionizing radiation tolerance of CMOS pixel sensors using a $0.18 \mu \mathrm{m}$ CMOS process, Nucl. Instrum. Meth. A831 (2016) 99-104, http://dx.doi.org/10.1016/j.nima.2016.03.031.

[6] L. Chen, et al., Characterization of the first prototype CMOS pixel sensor developed for the CEPC vertex detector, Radiat. Detect. Technol. Methods 3 (2019) 45, http://dx.doi.org/10.1007/s41605-019-0124-0. 
[7] Y. Han, et al., Test beam measurements of an irradiated prototype pixel sensor designed for the CEPC vertex detector, Nucl. Instrum. Meth. 977 (2020) 164267, http://dx.doi.org/10.1016/j.nima.2020.164267.

[8] I. Perić, C. Kreidl, P. Fischer, Particle pixel detectors in high-voltage CMOS technology - New achievements, Nucl. Instrum. Meth. 650 (1) (2011) 158-162, http://dx.doi.org/10.1016/j.nima.2010.11.090.

[9] V. Fadeyev, et al., Investigation of HV/HR-CMOS technology for the ATLAS phase-II strip tracker upgrade, Nucl. Instrum. Meth. 831 (2016) 189-196, http: //dx.doi.org/10.1016/j.nima.2016.05.092.

[10] B.T. Huffman, et al., Radiation hardness of two CMOS prototypes for the ATLAS HL-LHC upgrade project, J. Instrum. 11 (02) (2016) C02005, http://dx.doi.org/ 10.1088/1748-0221/11/02/c02005.
[11] K. Kanisauskas, et al., Radiation hardness studies of AMS HV-CMOS $350 \mathrm{~nm}$ prototype chip hvstripv1, J. Instrum. 12 (02) (2017) P02010, http://dx.doi.org/ 10.1088/1748-0221/12/02/p02010.

[12] B. Hiti, et al., Charge collection studies in irradiated HV-CMOS particle detectors, J. Instrum. 11 (04) (2016) P04007, http://dx.doi.org/10.1088/1748-0221/11/ 04/p04007.

[13] Z. Liang, et al., Study of built-in amplifier performance on HV-CMOS sensor for the ATLAS phase-II strip tracker upgrade, Nucl. Instrum. Meth. A831 (2016) 156-160, http://dx.doi.org/10.1016/j.nima.2016.05.007.

[14] C. Tamma, et al., The CHESS-2 prototype in AMS $0.35 \mu \mathrm{m}$ process: A high voltage CMOS monolithic sensor for ATLAS upgrade, 2016, http://dx.doi.org/10.1109/ NSSMIC.2016.8069856. 